\title{
Papers
}

\section{Methods of hysterectomy: systematic review and meta-analysis of randomised controlled trials}

\author{
Neil Johnson, David Barlow, Anne Lethaby, Emma Tavender, Liz Curr, Ray Garry
}

\begin{abstract}
Objective To evaluate the most appropriate surgical method of hysterectomy (abdominal, vaginal, or laparoscopic) for women with benign disease.

Design Systematic review and meta-analysis.

Data sources Cochrane Menstrual Disorders and Subfertility Group Trials Register, Cochrane Central Register of Controlled Trials, Medline, Embase, and Biological Abstracts.

Selection of studies Only randomised controlled trials were selected; participants had to have benign gynaecological disease; interventions had to comprise at least one hysterectomy method compared with another; and trials had to report primary outcomes (time taken to return to normal activities, intraoperative visceral injury, and major long term complications) or secondary outcomes (operating time, other immediate complications of surgery, short term complications, and duration of hospital stay).

Results 27 trials (total of 3643 participants) were included. Return to normal activities was quicker after vaginal than after abdominal hysterectomy (weighted mean difference 9.5 (95\% confidence interval 6.4 to 12.6) days) and after laparoscopic than after abdominal hysterectomy (difference 13.6 (11.8 to 15.4) days), but was not significantly different for laparoscopic versus vaginal hysterectomy (difference -1.1 ( -4.2 to 2.1 ) days). There were more urinary tract injuries with laparoscopic than with abdominal hysterectomy (odds ratio 2.61 (95\% confidence interval 1.22 to 5.60)), but no other intraoperative visceral injuries showed a significant difference between surgical approaches. Data were notably absent for many important long term patient outcome measures, where the analyses were underpowered to detect important differences, or they were simply not reported in trials.

Conclusions Significantly speedier return to normal activities and other improved secondary outcomes (shorter duration of hospital stay and fewer unspecified infections or febrile episodes) suggest that vaginal hysterectomy is preferable to abdominal hysterectomy where possible. Where vaginal hysterectomy is not possible, laparoscopic hysterectomy is preferable to abdominal hysterectomy, although it brings a higher chance of bladder or ureter injury.
\end{abstract}

\section{Introduction}

Three main types of hysterectomy are now used-abdominal, vaginal, and laparoscopic. Traditionally, abdominal hysterectomy has been used for gynaecological malignancy-when other pelvic disease is present, such as endometriosis or adhesions-or if the uterus is enlarged. It remains the "fallback option" if the uterus cannot be removed by another approach.

Vaginal hysterectomy was originally used only for prolapse, but it is now also used for menstrual abnormalities when the uterus is of fairly normal size. Vaginal hysterectomy is regarded as less invasive than abdominal hysterectomy.

In laparoscopic hysterectomy, at least part of the operation is done laparoscopically; this method requires greater surgical expertise than the vaginal and abdominal methods. The proportion of hysterectomies performed laparoscopically has gradually increased, and, although the procedure takes longer, proponents have emphasised several advantages: the opportunity to diagnose and treat other pelvic diseases (such as endometriosis) and to carry out adnexal surgery including the removal of the ovaries; the ability to secure thorough intraperitoneal haemostasis at the end of the procedure; and a rapid recovery time. ${ }^{2}$

Three subcategories of laparoscopic hysterectomy have been described. $^{3}$ In laparoscopic assisted vaginal hysterectomy $(\mathrm{LAVH})$, the procedure is done partly laparoscopically and partly vaginally, but the laparoscopic component does not involve uterine vessel ligation. In uterine vessel ligation laparoscopic hysterectomy ( $\mathrm{LH}(\mathrm{a})$ ), although the uterine vessels are ligated laparoscopically, part of the operation is done vaginally. In total laparoscopic hysterectomy, the entire operation (including suturing of the vaginal vault) is done laparoscopically. This method of laparoscopic hysterectomy requires the highest degree of surgical skill and is currently done only by a very small proportion of gynaecologists. It has been unclear whether total laparoscopic hysterectomy offers benefits over other forms of hysterectomy.

We subcategorised laparoscopic hysterectomy because surgeons using these methods need evidence based information about the particular procedure that they use.

The introduction of laparoscopic approaches in hysterectomies has prompted a much greater interest in the proper scientific evaluation of all forms of hysterectomy. This review aims to assess the most beneficial and least harmful surgical method.

\section{Methods}

In March 2004 we searched the Cochrane Menstrual Disorders and Subfertility Group Trials Register, the Cochrane Central Register of Controlled Trials, Medline, Embase, and Biological Abstracts. We performed data extraction and quantitative data synthesis according to the Cochrane Menstrual Disorders and Subfertility Group's guidelines. ${ }^{4}$ We selected trials according to the following eligibility criteria: we selected only randomised controlled trials; participants had to have benign gynaecological disease; interventions had to comprise at least one surgical 
approach to hysterectomy compared with another (excluding subtotal hysterectomy); and trials had to report primary outcomes (time it took participants to return to normal activities, intraoperative visceral injury, and major long term complications) or secondary outcomes (operating time, other immediate complications of surgery, short term complications, and duration of hospital stay).

We performed sensitivity analyses to examine the stability of the results in relation to "surgeon effect" and subcategorisation of laparoscopic hysterectomy.

\section{Results}

\section{Trial flow}

We identified 42 trials, of which we included $27^{5-31}$ (table 1) and excluded $10^{32-41}$ (with reasons for exclusion outlined in the longer version of this article, published as a Cochrane review ${ }^{4}$ ); the five remaining studies await assessment. ${ }^{42-46}$ We contacted the first authors of nine published abstracts to extract details that had not been reported: five studies were subsequently published, of which two were included ${ }^{6} 18$ and three excluded ${ }^{37} 3940$; four replies have not yet been received ${ }^{424346}$; and a further study, in Swedish, awaits translation. ${ }^{44}$

Of the 27 included trials (with a total of 3643 participants), two compared vaginal with abdominal hysterectomy, ${ }^{5}{ }^{18} 16$ compared laparoscopic with abdominal hysterectomy, ${ }^{7-9} 11131416171922$ 24-26 29-31 four compared laparoscopic with vaginal hysterectomy, ${ }^{6} 232728$ one compared LAVH with LH(a), ${ }^{15}$ one compared laparoscopic with abdominal and vaginal hysterectomy, ${ }^{10}$ and three compared laparoscopic, vaginal, and abdominal hysterectomy. ${ }^{12} 2021$

\section{Study characteristics and validity assessment}

Quality criteria, presented in detail elsewhere, ${ }^{4}$ are summarised in table 1 . The possibility that trial authors might have selectively reported "interesting" results potentially jeopardises the reliability of conclusions both from the individual studies and from this review.

We used Richardson's classification ${ }^{23}$ to categorise 22 of the 25 included studies that involved laparoscopic hysterectomy according to the amount of laparoscopic content (table 2). We also subcategorised these 22 trials as either LAVH, LH(a) or total, depending on the extent of the surgery performed laparoscopically and vaginally (table 1 ).

\section{Meta-analysis results}

All meta-analysis graphs, with summary statistics and 95\% confidence intervals for all comparisons and outcomes, are published electronically in the Cochrane Library. ${ }^{4}$

\section{Primary outcomes}

Return to normal activities-The meta-analysis in figure 1 shows that patients returned to normal activities sooner after vaginal than after abdominal hysterectomy (weighted mean difference 12.3 (95\% confidence interval 4.8 to 19.9 ) days); although statistical heterogeneity was present for this outcome (P value $0.02, \chi^{2}$ test; $\mathrm{I}^{2}=75.3 \%$ ), similar results were obtained with both fixed and random effects models. Return to normal activities was also quicker after laparoscopic than after abdominal hysterectomy (difference 13.3 (9.9 to 16.8) days); although statistical heterogeneity was present $\left(\mathrm{P}\right.$ value $0.004, \chi^{2}$ test; $\left.\mathrm{I}^{2}=71.2 \%\right)$, similar results were obtained using both fixed and random effects models. We found no significant difference between laparoscopic and vaginal hysterectomy in return to normal activities $(-1.1$ ( -4.2 to 2.1$)$ days).
Intraoperative visceral injury-Where bladder and ureter injuries were pooled as "urinary tract injury" (figure 2), we found a significant increase in urinary tract injury for laparoscopic versus abdominal hysterectomy (odds ratio 2.61 (95\% confidence interval 1.22 to 5.60$)$ ) but no significant differences in urinary tract injury for laparoscopic versus vaginal hysterectomy (1.00 (0.36 to 2.75$)$ ) or for LH(a) versus LAVH (1.60 (0.29 to 7.83)). No other intraoperative visceral injuries (including bladder and ureter considered independently, and bowel and vascular injury) showed a significant difference between surgical approaches.

Major long term complications -We found no significant differences in fistula formation, urinary dysfunction, sexual dysfunction, or patient satisfaction when we compared surgical approaches, although for most of these outcomes the analyses were underpowered to detect important differences. Data were notably not reported in trials for many important long term outcome measures, including chronic pelvic or abdominal pain, bowel dysfunction, and vaginal prolapse.

\section{Secondary outcomes}

Operation time-Both trials in the meta-analysis of vaginal versus abdominal hysterectomy showed a significant difference in the length of time of the operation, but in opposite directions. Abdominal hysterectomies were performed significantly faster than laparoscopic hysterectomies (weighted mean difference 18.0 (95\% confidence interval 1.0 to 35.1 ) minutes), although this difference was not apparent in trials where the subcategory LAVH was compared with abdominal hysterectomy. Statistical heterogeneity was present for operation time for laparoscopic versus abdominal hysterectomy $\left(\mathrm{P}\right.$ value $<0.0001, \chi^{2}$ test; $\mathrm{I}^{2}=96.2 \%$ ), but similar results were obtained with fixed and random effects models, except for a significantly shorter operation time for the LAVH subcategory versus abdominal hysterectomy, apparent with a fixed effects model (difference 7.6 (3.0 to 12.2) minutes). Vaginal hysterectomy also had a shorter operation time than laparoscopic hysterectomy (difference 44.5 (26.2 to 62.8) minutes), and, although statistical heterogeneity was present ( $\mathrm{P}$ value $0.001, \chi^{2}$ test; $\mathrm{I}^{2}=80.6 \%$ ), similar results were obtained with fixed and random effects models. LAVH had a significantly shorter operation time than $\mathrm{LH}(\mathrm{a})$ (difference 25.3 (10.0 to 40.6) minutes).

Other intraoperative complications-The number of women with substantial bleeding and the incidence of unintended laparotomy (where abdominal hysterectomy was not one of the treatment comparisons) did not differ significantly between surgical approaches.

Short term outcomes and complications-Hospital stay was significantly shorter for women who had had vaginal rather than abdominal hysterectomy (weighted mean difference 1.0 (0.7 to 1.2) days) or laparoscopic rather than abdominal hysterectomy (difference 2.0 (1.9 to 2.2) days); statistical heterogeneity was present ( $\mathrm{P}$ value $<0.0001, \chi^{2}$ test; $\mathrm{I}^{2}=95.0 \%$ ), but similar results were obtained with a random effects model. Duration of hospital stay was not significantly different for laparoscopic versus vaginal hysterectomy or for $\mathrm{LH}(\mathrm{a})$ versus LAVH. For vaginal versus abdominal hysterectomy, there were significantly fewer unspecified infections or febrile episodes (odds ratio $0.42 \quad(95 \%$ confidence interval 0.21 to 0.83 )). For laparoscopic versus abdominal hysterectomy, there were significantly fewer wound or abdominal wall infections (0.32 (0.12 to 0.85$))$ and significantly fewer unspecified infections or febrile episodes $(0.65$ ( 0.49 to $0.87)$ ). There were no significant differences between surgical approaches in the need for blood transfusion, although laparoscopic hysterectomy was associated with a significantly 
Table 1 Quality of studies included in meta-analysis

\begin{tabular}{clll} 
Trial (comparison) & $\begin{array}{l}\text { Randomisation (and } \\
\text { allocation concealment) }\end{array}$ & $\begin{array}{l}\text { Single centre or } \\
\text { multicentre }\end{array}$ & $\begin{array}{l}\text { Power calculation } \\
\text { (and sample size) }\end{array}$ \\
\hline $\begin{array}{c}\text { Benassi, 2002 } \\
\text { (VH } v \text { AH) }\end{array}$ & $\begin{array}{l}\text { Computer generated } \\
\text { (unclear) }\end{array}$ & Single & None $(\mathrm{n}=119)$ \\
& & & \\
\end{tabular}

\begin{tabular}{|c|c|c|c|c|c|}
\hline $\begin{array}{l}\text { Miskry, } 2003^{18} \\
\text { (VH } v \text { AH) }\end{array}$ & $\begin{array}{l}\text { Computer generated (sealed } \\
\text { opaque envelopes) }\end{array}$ & $\begin{array}{l}\text { Multicentre } \\
\text { ( } 2 \text { centres) }\end{array}$ & $\begin{array}{l}36 \text { participants } \\
\text { required; primary } \\
\text { outcome: } \\
\text { hospital stay }(n=36)\end{array}$ & $\begin{array}{l}\text { Excluded uterine size }>14 \text { weeks, } \\
\text { malignancy, adnexal pathology, } \\
\text { reduced uterine mobility, reduced } \\
\text { vaginal access, need for prolapse } \\
\text { or incontinence surgery }\end{array}$ & $\begin{array}{l}\text { Short term outcomes (transfusion, pelvic haematoma, } \\
\text { abdominal wound infection, UTI, febrile morbidity); } \\
\text { recovery (hospital stay, return to normal) }\end{array}$ \\
\hline $\begin{array}{l}\text { Ellstrom, } 1998^{7} \\
\quad(\text { LH } v \text { AH) }\end{array}$ & Not reported (unclear) & Single & None $(n=40)$ & $\begin{array}{l}\text { Included women due for AH or in } \\
\text { whom VH contraindicated }\end{array}$ & $\begin{array}{l}\text { Operating time; immediate complications; short term } \\
\text { outcomes (transfusion, vaginal cuff infection, febrile } \\
\text { morbidity) }\end{array}$ \\
\hline $\begin{array}{l}\text { Falcone, } 1999^{8} \\
\quad(\mathrm{LH} \vee \mathrm{AH})\end{array}$ & $\begin{array}{l}\text { Computer generated } \\
\text { (unclear) }\end{array}$ & Single & $\begin{array}{l}44 \text { participants } \\
\text { required; primary } \\
\text { outcome: operation } \\
\text { time }(n=48)\end{array}$ & $\begin{array}{l}\text { Included women due for AH or in } \\
\text { whom VH contraindicated }\end{array}$ & $\begin{array}{l}\text { Operating time; immediate complications (blood loss, } \\
\text { intraoperative complications); short term outcomes } \\
\text { (pain, vaginal cuff infection, UTI, chest infection, febrile } \\
\text { morbidity); recovery (hospital stay, return to normal) }\end{array}$ \\
\hline $\begin{array}{l}\text { Ferrari, } 2000^{9} \\
(\text { LH } v \text { AH) }\end{array}$ & $\begin{array}{l}\text { Computer generated (sealed } \\
\text { opaque envelopes) }\end{array}$ & Single & None $(\mathrm{n}=62)$ & $\begin{array}{l}\text { Included women due for AH or in } \\
\text { whom VH contraindicated and } \\
\text { large fibroid uterus }\end{array}$ & $\begin{array}{l}\text { Operating time; immediate complications (blood loss, } \\
\text { intraoperative complications); short term outcomes } \\
\text { (transfusion, febrile morbidity, analgesic requirement); } \\
\text { recovery (hospital stay) }\end{array}$ \\
\hline $\begin{array}{l}\text { Härkki-Sirén } 2000^{11} \\
\text { (LH v AH) }\end{array}$ & $\begin{array}{l}\text { Not reported (sealed opaque } \\
\text { envelopes) }\end{array}$ & Single & $\begin{array}{l}42 \text { participants } \\
\text { required; primary } \\
\text { outcome: } C \text { reactive } \\
\text { protein level }(n=50)\end{array}$ & $\begin{array}{l}\text { Included women due for AH or in } \\
\text { whom VH contraindicated }\end{array}$ & $\begin{array}{l}\text { Operating time; immediate complications (blood loss); } \\
\text { short term outcomes (Hb change, vaginal cuff infection, } \\
\text { abdominal wound infection); recovery (hospital stay, } \\
\text { return to normal) }\end{array}$ \\
\hline $\begin{array}{r}\text { Kunz, } 1996^{13} \\
\text { (LH } \vee \text { AH) }\end{array}$ & Not reported (unclear) & Single & None $(n=70)$ & $\begin{array}{l}\text { Included women scheduled for } \\
\text { hysterectomy for non-malignant } \\
\text { disease }\end{array}$ & $\begin{array}{l}\text { Operating time; immediate complications; short term } \\
\text { outcomes (Hb change, pain relief); recovery (hospital } \\
\text { stay) }\end{array}$ \\
\hline $\begin{array}{l}\text { Langebrekke } 1996^{14} \\
(\mathrm{LH} v \mathrm{AH})\end{array}$ & $\begin{array}{l}\text { Random number table } \\
\text { (sealed opaque envelopes) }\end{array}$ & $\begin{array}{l}\text { Multicentre } \\
\text { ( } 2 \text { centres) }\end{array}$ & None $(n=100)$ & $\begin{array}{l}\text { Excluded malignancy, suspected } \\
\text { adhesions, }>12 \text { week uterus, } \\
\text { cardiopulmonary disease, } \\
\text { previous colporrhaphy }\end{array}$ & $\begin{array}{l}\text { Operating time; immediate complications (blood loss, } \\
\text { bladder and ureter injury); short term outcomes (pain, } \\
\text { pelvic haematoma, abdominal wound infection); recovery } \\
\text { (hospital stay) }\end{array}$ \\
\hline $\begin{array}{l}\text { Lumsden, } 2000^{16} \\
\quad(\mathrm{LH} v \mathrm{AH})\end{array}$ & $\begin{array}{l}\text { Computer generated } \\
\text { (unclear) }\end{array}$ & $\begin{array}{l}\text { Multicentre } \\
\text { (3 centres) }\end{array}$ & $\begin{array}{l}240 \text { participants } \\
\text { required; primary } \\
\text { outcome: } \\
\text { complications }(n=200)\end{array}$ & $\begin{array}{l}\text { Included women due for AH or in } \\
\text { whom VH contraindicated }\end{array}$ & $\begin{array}{l}\text { Operating time; immediate complications (major- } \\
\text { including ureter and bowel injury-and minor); short } \\
\text { term outcomes (ITU admission, blood transfusion, } \\
\text { reoperation, abdominal wound infection, UTI, chest } \\
\text { infection, febrile morbidity, thromboembolism); recovery } \\
\text { (hospital stay); long term outcomes (urinary } \\
\text { dysfunction, satisfaction) }\end{array}$ \\
\hline $\begin{array}{l}\text { Marana, } 1999^{17} \\
\text { (LH } \vee \text { AH) }\end{array}$ & $\begin{array}{l}\text { Computer generated } \\
\text { (unclear) }\end{array}$ & $\begin{array}{l}\text { Multicentre } \\
\text { (4 centres) }\end{array}$ & $\begin{array}{l}116 \text { participants } \\
\text { required; primary } \\
\text { outcome: } \\
\text { complications }(n=116)\end{array}$ & $\begin{array}{l}\text { Included women due for AH or in } \\
\text { whom VH contraindicated }\end{array}$ & $\begin{array}{l}\text { Operating time; immediate complications (blood loss, } \\
\text { laparotomy conversion, bladder injury); short term } \\
\text { outcomes (postoperative complications, pain, Hb drop, } \\
\text { transfusion, pelvic haematoma, vaginal cuff infection, } \\
\text { febrile morbidity); recovery (hospital stay) }\end{array}$ \\
\hline $\begin{array}{l}\text { Olsson, } 1996^{19} \\
\text { (LH v AH) }\end{array}$ & $\begin{array}{l}\text { Not reported (sealed opaque } \\
\text { envelopes) }\end{array}$ & Single & $\begin{array}{l}140 \text { participants } \\
\text { needed; primary } \\
\text { outcome: } \\
\text { complications ( } n=143 \text { ) }\end{array}$ & $\begin{array}{l}\text { Specifically included women due } \\
\text { for AH or in whom VH } \\
\text { contraindicated }\end{array}$ & $\begin{array}{l}\text { Operating time; immediate complications (blood loss, } \\
\text { bladder injury); short term outcomes (pain relief, } \\
\text { transfusion, pelvic haematoma, vaginal cuff infection, } \\
\text { UTI, febrile morbidity); recovery (hospital stay, return to } \\
\text { normal); long term outcomes (fistula) }\end{array}$ \\
\hline $\begin{array}{l}\text { Perino, } 1999^{21} \\
\text { (LH v AH) }\end{array}$ & Not reported (unclear) & Single & None $(n=102)$ & $\begin{array}{l}\text { Scheduled hysterectomy for } \\
\text { non-malignant disease }\end{array}$ & $\begin{array}{l}\text { Operating time; immediate complications (blood loss, } \\
\text { ureter injury); short term outcomes (Hb drop, pain, } \\
\text { complications including febrile morbidity); recovery } \\
\text { (hospital stay); long term outcomes (fistula) }\end{array}$ \\
\hline $\begin{array}{l}\text { Raju, } 1994^{22} \text { (LH } v \\
\text { AH) }\end{array}$ & $\begin{array}{l}\text { Computer generated (sealed } \\
\text { opaque envelopes) }\end{array}$ & Single & $\begin{array}{l}80 \text { participants } \\
\text { needed; primary } \\
\text { outcome, morbidity } \\
(\mathrm{n}=80)\end{array}$ & $\begin{array}{l}\text { Compared LH (BSO) with } \mathrm{AH} \\
\text { (BSO) }\end{array}$ & $\begin{array}{l}\text { Operating time; immediate complications (blood loss, } \\
\text { vascular injury); short term outcomes (Hb drop, pain } \\
\text { pelvic haematoma); recovery (hospital stay, return to } \\
\text { normal) }\end{array}$ \\
\hline $\begin{array}{l}\text { Schutz, } 2002^{25} \text { (LH v } \\
\text { AH) }\end{array}$ & $\begin{array}{l}\text { Computer generated } \\
\text { (telephone inquiry to third } \\
\text { party) }\end{array}$ & Single & $\begin{array}{l}\text { Power calculation } \\
\text { performed }(n=48)\end{array}$ & $\begin{array}{l}\text { Included women with large } \\
\text { uterus }\end{array}$ & $\begin{array}{l}\text { Operating time; immediate complications (blood loss); } \\
\text { short term outcomes (pain, transfusion, UTI); recovery } \\
\text { (hospital stay, return to normal) }\end{array}$ \\
\hline $\begin{array}{l}\text { Seracchioli, } 2002^{26} \\
\text { (LH } v \text { AH) }\end{array}$ & $\begin{array}{l}\text { Computer generated } \\
\text { (unclear) }\end{array}$ & Single & None $(n=122)$ & $\begin{array}{l}\text { Included women due for AH or in } \\
\text { whom VH contraindicated }\end{array}$ & $\begin{array}{l}\text { Operating time; immediate complications (blood loss, } \\
\text { laparoconversion); short term outcomes (Hb drop, } \\
\text { transfusion, febrile morbidity); recovery (hospital stay, } \\
\text { return to normal) }\end{array}$ \\
\hline $\begin{array}{l}\text { Summitt, } 1998^{29} \text { (LH V } \\
\text { AH) }\end{array}$ & $\begin{array}{l}\text { Computer generated (sealed } \\
\text { opaque envelopes) }\end{array}$ & $\begin{array}{l}\text { Multicentre (3 } \\
\text { centres) }\end{array}$ & None $(n=67)$ & $\begin{array}{l}\text { Specifically included women due } \\
\text { for } \mathrm{AH} \text { or in whom VH } \\
\text { contraindicated }\end{array}$ & $\begin{array}{l}\text { Operating time; immediate complications (blood loss, } \\
\text { laparoconversion, intraoperative complications; short } \\
\text { term outcomes (pain, transfusion, fever, abdominal } \\
\text { wound infection); recovery (hospital stay, return to } \\
\text { normal) }\end{array}$ \\
\hline Tsai, $2003^{30}(\mathrm{LH} v \mathrm{AH})$ & $\begin{array}{l}\text { Computer generated } \\
\text { (unclear) }\end{array}$ & Single & None $(n=200)$ & $\begin{array}{l}\text { Included women due for AH or in } \\
\text { whom VH contraindicated and } \\
\text { large fibroid uterus }\end{array}$ & $\begin{array}{l}\text { Operating time; immediate complications (bladder } \\
\text { injury); short term outcomes (transfusion, vaginal cuff } \\
\text { infection); recovery (hospital stay) }\end{array}$ \\
\hline $\begin{array}{l}\text { Yuen, } 1998^{31} \text { (LH v } \\
\text { AH) }\end{array}$ & $\begin{array}{l}\text { Computer generated } \\
\text { (unclear) }\end{array}$ & Single & None $(n=50)$ & $\begin{array}{l}\text { Included women due for AH or in } \\
\text { whom VH contraindicated }\end{array}$ & $\begin{array}{l}\text { Operating time; immediate complications (blood loss); } \\
\text { short term outcomes (transfusion, pelvic haematoma, } \\
\text { abdominal wound infection, UTI, febrile morbidity); } \\
\text { recovery (hospital stay) }\end{array}$ \\
\hline
\end{tabular}

Inclusion and

\section{exclusion criteria}

Included large fibroid uterus;

excluded prolapse, vaginal

stenosis, neoplasia, previous

Full reporting of outcomes

Operating time; immediate complications (vessel, ureter, bladder, and bowel injury); short term outcomes ( $\mathrm{Hb}$ drop, transfusion, pelvic haematoma, abdominal wound drop, transfusion, pelvic haematoma, abdominal wound infection, febrile morbidity, thromboembolism); recover
(hospital stay); Iong term outcomes (satisfaction)

Short term outcomes (transfusion, pelvic haematoma, 


\begin{tabular}{|c|c|c|c|c|c|}
\hline Trial (comparison) & $\begin{array}{l}\text { Randomisation (and } \\
\text { allocation concealment) }\end{array}$ & $\begin{array}{l}\text { Single centre or } \\
\text { multicentre }\end{array}$ & $\begin{array}{l}\text { Power calculation } \\
\text { (and sample size) }\end{array}$ & $\begin{array}{l}\text { Inclusion and } \\
\text { exclusion criteria }\end{array}$ & Full reporting of outcomes \\
\hline $\begin{array}{l}\text { Darai, } 2001^{6} \\
\quad(\mathrm{LH} v \mathrm{VH})\end{array}$ & $\begin{array}{l}\text { Computer generated } \\
\text { (unclear) }\end{array}$ & $\begin{array}{l}\text { Multicentre } \\
\text { (2 centres) }\end{array}$ & $\begin{array}{l}70 \text { participants } \\
\text { required; primary } \\
\text { outcome: } \\
\text { complications }(n=80)\end{array}$ & Included uterine size $>280 \mathrm{~g}$ & $\begin{array}{l}\text { Operating time; immediate complications } \\
\text { (laparoconversion, bladder and vascular injury); short } \\
\text { term outcomes (pain, Hb drop, transfusion, pelvic } \\
\text { haematoma, vaginal cuff infection, wound infection, } \\
\text { febrile morbidity); recovery (hospital stay) }\end{array}$ \\
\hline $\begin{array}{l}\text { Richardson, } 1995^{23} \\
\quad(\mathrm{LH} v \mathrm{VH})\end{array}$ & $\begin{array}{l}\text { Random number table } \\
\text { (unclear) }\end{array}$ & Single & None $(n=45)$ & Excluded uterine size $>16$ weeks & $\begin{array}{l}\text { Operating time; immediate complications (bladder and } \\
\text { vascular injury, laparotomy); short term outcomes } \\
\text { (postoperative complications); recovery (hospital stay, } \\
\text { return to normal) }\end{array}$ \\
\hline $\begin{array}{l}\text { Soriano, } 2001^{27} \\
\quad(\mathrm{LH} \vee \mathrm{VH})\end{array}$ & $\begin{array}{l}\text { Computer generated } \\
\text { (unclear) }\end{array}$ & Single & $\begin{array}{l}70 \text { participants } \\
\text { required; primary } \\
\text { outcome: } \\
\text { complications }(n=80)\end{array}$ & Included uterine size $>280 \mathrm{~g}$ & $\begin{array}{l}\text { Operating time; immediate complications (blood loss, } \\
\text { laparotomy; short term outcomes (Hb drop, transfusion, } \\
\text { pain, postoperative complications); recovery (hospital stay) }\end{array}$ \\
\hline $\begin{array}{l}\text { Summitt, } 1992^{28} \\
\text { (LH } v \text { VH) }\end{array}$ & $\begin{array}{l}\text { Computer generated } \\
\text { (unclear) }\end{array}$ & Single & None $(n=56)$ & Excluded uterine size $>16$ weeks & $\begin{array}{l}\text { Operating time; immediate complications (blood loss, } \\
\text { laparotomy, bladder and vascular injury); short term } \\
\text { outcomes (pain, vaginal cuff infection, febrile morbidity); } \\
\text { long term outcomes (fistula) }\end{array}$ \\
\hline $\begin{array}{l}\text { Garry, 2004 } \\
\quad(\mathrm{LH} \vee \mathrm{AH}, \mathrm{LH} \vee \mathrm{VH})\end{array}$ & $\begin{array}{l}\text { Computer generated } \\
\text { (telephone inquiry) }\end{array}$ & $\begin{array}{l}\text { Multicentre } \\
\text { (30 centres) }\end{array}$ & $\begin{array}{l}\text { Power calculation with } \\
\text { primary outcome } \\
\text { major complications; } \\
\text { power achieved in LH } \\
V \text { AH arm, not in LH v } \\
\text { VH arm }(n=1380)\end{array}$ & $\begin{array}{l}\text { Excluded uterine size }>12 \text { weeks, } \\
\text { suspected malignancy, prolapse, } \\
\text { serious medical illness, need for } \\
\text { pelvic support surgery }\end{array}$ & $\begin{array}{l}\text { Operating time; major and minor complications; immediate } \\
\text { complications (haemorrhage, bowel, bladder, ureter and } \\
\text { vascular injury, unintended laparotomy); short term } \\
\text { outcomes (transfusion, PE, wound dehiscence, haematoma, } \\
\text { pyrexia, infection, DVT, pain); recovery (hospital stay); } \\
\text { long term outcomes (quality of life, body image) }\end{array}$ \\
\hline $\begin{array}{l}\text { Hwang, } 2002^{12} \\
\quad(\text { LH } v \text { VH } v \text { AH) }\end{array}$ & $\begin{array}{l}\text { Computer generated (sealed } \\
\text { opaque envelopes) }\end{array}$ & Single & $\begin{array}{l}\text { Retrospective power } \\
\text { calculation }(n=90)\end{array}$ & $\begin{array}{l}\text { Included large fibroid uterus; } \\
\text { excluded adenomyosis, prolapse, } \\
\text { chronic pelvic pain, DUB, cervical } \\
\text { dysplasia, PID }\end{array}$ & $\begin{array}{l}\text { Operating time; immediate complications (blood loss, } \\
\text { intraoperative complications); short term outcomes } \\
\text { (tenderness, transfusion, pelvic haematoma, vaginal cuff } \\
\text { infection, UTI, chest infection, febrile morbidity); } \\
\text { recovery (hospital stay, return to normal) }\end{array}$ \\
\hline $\begin{array}{l}\text { Ottosen, } 2000^{20} \\
\quad(\text { LH } v \text { VH } \vee \text { AH) }\end{array}$ & $\begin{array}{l}\text { Computer generated (sealed } \\
\text { opaque envelopes) }\end{array}$ & Single & $\begin{array}{l}40 \text { participants } \\
\text { required; primary } \\
\text { outcome: duration of } \\
\text { hospital stay }(n=120)\end{array}$ & $\begin{array}{l}\text { Excluded uterine size }>16 \text { weeks, } \\
\text { adhesions, narrow vagina, or } \\
\text { inaccessible uterus }\end{array}$ & $\begin{array}{l}\text { Operating time; immediate complications (blood loss, } \\
\text { bladder injury, unintended laparotomy); short term } \\
\text { outcomes (transfusion, pelvic haematoma, vaginal cuff } \\
\text { infection, UTI, febrile morbidity); recovery (hospital stay, } \\
\text { return to normal); long term outcomes (urinary } \\
\text { dysfunction) }\end{array}$ \\
\hline $\begin{array}{l}\text { Ribiero, } 2003^{24} \\
\quad(\text { LH } v \text { VH } v \text { AH) }\end{array}$ & Not reported (unclear) & Single & None $(n=60)$ & $\begin{array}{l}\text { Included large fibroid uterus; } \\
\text { excluded }>400 \text { ml uterus, NSAID } \\
\text { users, diabetes mellitus, } \\
\text { coagulation disorders, } \\
\text { autoimmune disease }\end{array}$ & $\begin{array}{l}\text { Operating time; immediate complications (bladder } \\
\text { injury); short term outcomes ( } \mathrm{Hb} \text { change) }\end{array}$ \\
\hline $\begin{array}{l}\text { Long, 2001 } \\
\text { (LH(a) } v \text { LAVH) }\end{array}$ & Not reported (unclear) & Single & None $(n=167)$ & $\begin{array}{l}\text { Included contraindications for VH, } \\
\text { large fibroid uterus, previous } \\
\text { pelvic surgery, PID, need for } \\
\text { adnexectomy, lack of uterine } \\
\text { descent, and limited vaginal } \\
\text { access; excluded }>16 \text { week uterus }\end{array}$ & $\begin{array}{l}\text { Operating time; immediate complications (blood loss, } \\
\text { laparotomy, bladder, ureter, bowel and vascular injury); } \\
\text { short term outcomes (transfusion, vaginal cuff infection, } \\
\text { febrile morbidity); recovery (hospital stay); Iong term } \\
\text { outcomes (sexual dysfunction) }\end{array}$ \\
\hline
\end{tabular}

Blinding was not reported in any of the included trials.

Most trials had no drop-outs. Exceptions were Falcone ${ }^{8}$ (7 drop-outs preoperatively; intention to treat (ITT) reported); Lumsden ${ }^{16}$ (10 drop-outs; no ITT); Summitt ${ }^{29}$ (2 drop-outs; no ITT); Yuen ${ }^{31}$ (6 drop-outs; no ITT); Garry ${ }^{10}$ (45 drop-outs preoperatively; ITT reported); Long ${ }^{15}$ (13 drop-outs; exclusion of further 53 participants; no ITT). Tsai ${ }^{30}$ had no drop-outs, but 2 cases were not analysed. For information about surgeon effect, see the longer version of this paper (published as a Cochrane review ${ }^{4}$ ).

$\mathrm{AH}=$ abdominal hysterectomy; $\mathrm{VH}=$ =vaginal hysterectomy; $\mathrm{LH}=$ laparoscopic hysterectomy; LAVH=laparoscopic assisted vaginal hysterectomy; $\mathrm{LH}(\mathrm{a})=$ laparoscopic hysterectomy in which laparoscopic procedure includes uterine artery ligation; $\mathrm{Hb}$ =haemoglobin; UTI=urinary tract infection; ITU=intensive therapy unit; $\mathrm{BSO}=\mathrm{bilateral} \mathrm{salpingo-00phorectomy;} \mathrm{DUB=dysfunctional} \mathrm{uterine} \mathrm{bleeding;}$

PID=pelvic inflammatory disease; NSAID=non-steroidal anti-inflammatory drug; PE=pulmonary embolism; DVT=deep vein thrombosis; HRT=hormone replacement therapy.

lower mean blood loss than abdominal hysterectomy (weighted mean difference $45.3 \mathrm{ml}(95 \%$ confidence interval $17.9 \mathrm{ml}$ to $72.7 \mathrm{ml}))$ and a smaller drop in haemoglobin $(0.55 \mathrm{~g} / \mathrm{l}(0.28 \mathrm{~g} / \mathrm{l}$ to $0.82 \mathrm{~g} / \mathrm{l})$ ). We found no evidence of a significant difference between surgical approaches for occurrence of pelvic haematoma, vaginal cuff infection, urinary tract infection, chest infection, or thromboembolic events.

\section{Sensitivity analyses}

Exclusion of the three trials in which surgeons for one intervention were unequivocally different from those performing the other intervention ${ }^{14}{ }^{22}$ did not alter the significance of any meta-analysis results.

When laparoscopic hysterectomy was subcategorised, the longer operating time compared with abdominal hysterectomy was not apparent for LAVH. All other subcategory metaanalyses of laparoscopic versus abdominal hysterectomy and laparoscopic versus vaginal hysterectomy showed results that were similar to the respective meta-analysis of laparoscopic hysterectomy as a pooled group.

\section{Data not included in meta-analysis}

Data expressed as medians were not included in the meta-analysis, and these results are presented in full elsewhere. ${ }^{4}$ These data showed consistently lower postoperative pain scores for laparoscopic than abdominal hysterectomy, in addition to improved quality of life, body image scores; and increased sexual frequency at six weeks, but these differences disappeared by one year. ${ }^{10}$ Mean total hospital cost was significantly higher for laparoscopic than vaginal hysterectomy. ${ }^{28}$

\section{Discussion}

Our data suggest that vaginal hysterectomy is preferable to abdominal hysterectomy, provided that it can be done safely. Claims that laparoscopic hysterectomy can allow identification of pelvic disease that might otherwise lead to complications during vaginal hysterectomy and that the meticulous haemostasis achievable during laparoscopic hysterectomy might reduce pelvic haematomas or vaginal cuff infections have not been 


\begin{tabular}{|c|c|c|}
\hline Stage & Laparoscopic content & Included trials \\
\hline 0 & $\begin{array}{l}\text { Laparoscopy performed but no } \\
\text { laparoscopic procedure before vaginal } \\
\text { hysterectomy }\end{array}$ & Ottosen ${ }^{20}$ \\
\hline 1 & $\begin{array}{l}\text { Procedure includes laparoscopic } \\
\text { adhesiolysis or excision of endometriosis }\end{array}$ & No trial \\
\hline 2 & $\begin{array}{l}\text { Either or both adnexae freed } \\
\text { laparoscopically }\end{array}$ & Kunz, ${ }^{13}$ Marana, ${ }^{17}$ Raju ${ }^{22}$ \\
\hline 3 & $\begin{array}{l}\text { Bladder dissected from uterus } \\
\text { laparoscopically }\end{array}$ & Ferrari, ${ }^{9}$ Long, ${ }^{15}$ Tsai $^{30}$ \\
\hline 4 & Uterine artery transected laparoscopically & $\begin{array}{l}\text { Darai, }{ }^{6} \text { Ellstrøm, }{ }^{7} \text { Olsson, }{ }^{19} \text { Schutzz, } \\
\text { Soriano, }{ }^{27} \text { Summitt, }{ }^{28} \text { Summitt, }{ }^{29} \text { Yuen }{ }^{31}\end{array}$ \\
\hline 5 & $\begin{array}{l}\text { Anterior or posterior colpotomy or entire } \\
\text { uterus freed laparoscopically }\end{array}$ & $\begin{array}{l}\text { Falcone }{ }^{8} \text { Härkki-Sirén },{ }^{11} \text { Hwang, }{ }^{12} \\
\text { Langebrekke }{ }^{14} \text { Long },{ }^{15} \text { Perino, }{ }^{21} \text { Ribiero, }{ }^{2}{ }^{2} \\
\text { Seracchioli }{ }^{26}\end{array}$ \\
\hline
\end{tabular}

Three trials ${ }^{11} 1724$ could not be categorised.

One trial ${ }^{15}$ compared stage 3 versus stage 5 laparoscopic hysterectomy.

Two trials ${ }^{22}{ }^{25}$ used total laparoscopic hysterectomy as an intervention (all of the surgical manipulation, including incision and suturing of the vaginal vault, being carried out

laparoscopically, even though the uterus was removed transvaginally).

borne out in this review. However, a laparoscopic approach may be appropriate if an oophorectomy is needed. Whether the increased detection of unexpected disease at laparoscopic hysterectomy (compared with vaginal hysterectomy) ${ }^{10}$ affects subsequent clinical outcomes remains uncertain.

\section{Operating time}

Operating time is longer for laparoscopic than for both abdominal and vaginal hysterectomy. However, LAVH had a significantly shorter operating time than abdominal hysterectomy, and LAVH had a significantly shorter mean operating time than $\mathrm{LH}(\mathrm{a})$, the latter being the lengthiest operation.

\section{Urinary tract injury}

The increased incidence of urinary tract injury (bladder and ureter injuries pooled as a single category) from laparoscopic hysterectomy seen in our review supports that reported elsewhere in non-randomised studies. ${ }^{10} 4788$ However, our study was not powerful enough to detect an increase in ureteric injury considered independently (that occurred in 1 in 78 women having laparoscopic hysterectomy and 1 in 492 women having abdominal hysterectomy). Urinary tract damage, in particular ureteric injury, remains the major concern in relation to the laparoscopic approach. Furthermore, in the largest randomised controlled trial included in this review ${ }^{10}$ the authors pooled cases in which at least one major complication occurred and found a

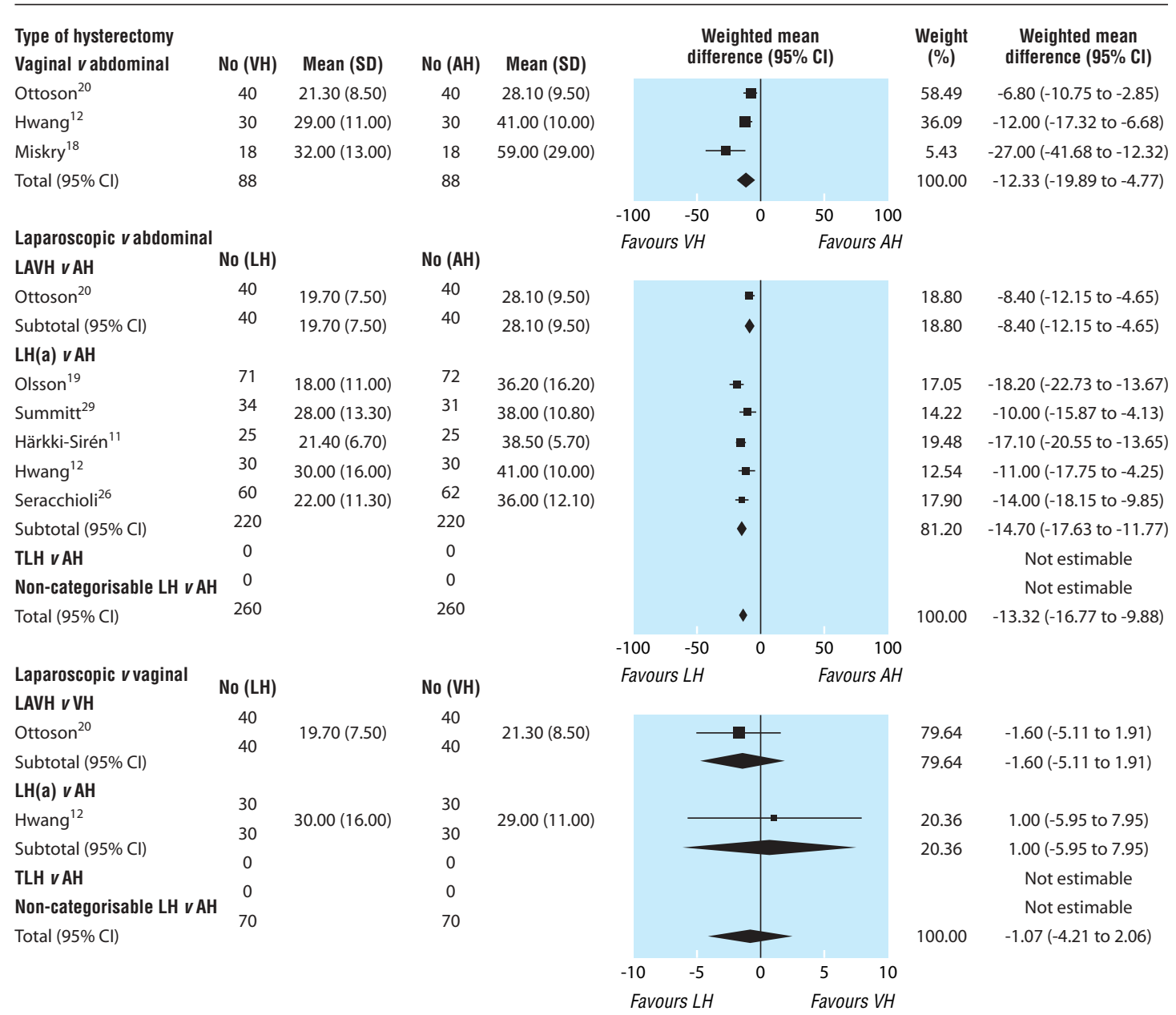

Figure 1 Meta-analysis of return to normal activities (number of days). Statistical pooling used random effects statistical model for vaginal versus abdominal hysterectomy and for laparoscopic versus abdominal hysterectomy, and fixed effects statistical model for laparoscopic versus vaginal hysterectomy. AH=abdominal hysterectomy; $\mathrm{VH}=$ vaginal hysterectomy; $\mathrm{LH}=$ laparoscopic hysterectomy; LAVH=laparoscopic assisted vaginal hysterectomy; $\mathrm{LH}(\mathrm{a})=$ laparoscopic hysterectomy where laparoscopic procedures include uterine artery ligation; TLH=total laparoscopic hysterectomy 
significant increase in this outcome for laparoscopic versus abdominal hysterectomy (but not laparoscopic versus vaginal hysterectomy). Although it could be speculated that laparoscopic uterine artery ligation is the manoeuvre most likely to increase the risk of ureteric injury, especially if the surgeon is unskilled in such a procedure, we were unable to confirm this as trials of LAVH versus abdominal hysterectomy did not report on ureteric injury.

\section{Methodological challenges and surgical training}

It is particularly difficult to assess validity of methodologies used in systematic reviews where surgical rather than medical interventions are tested-complicated by variable expertise among surgeons and the learning process. This is well illustrated by heterogeneity in such outcomes as operating time, even when the "traditional" vaginal and abdominal hysterectomy techniques

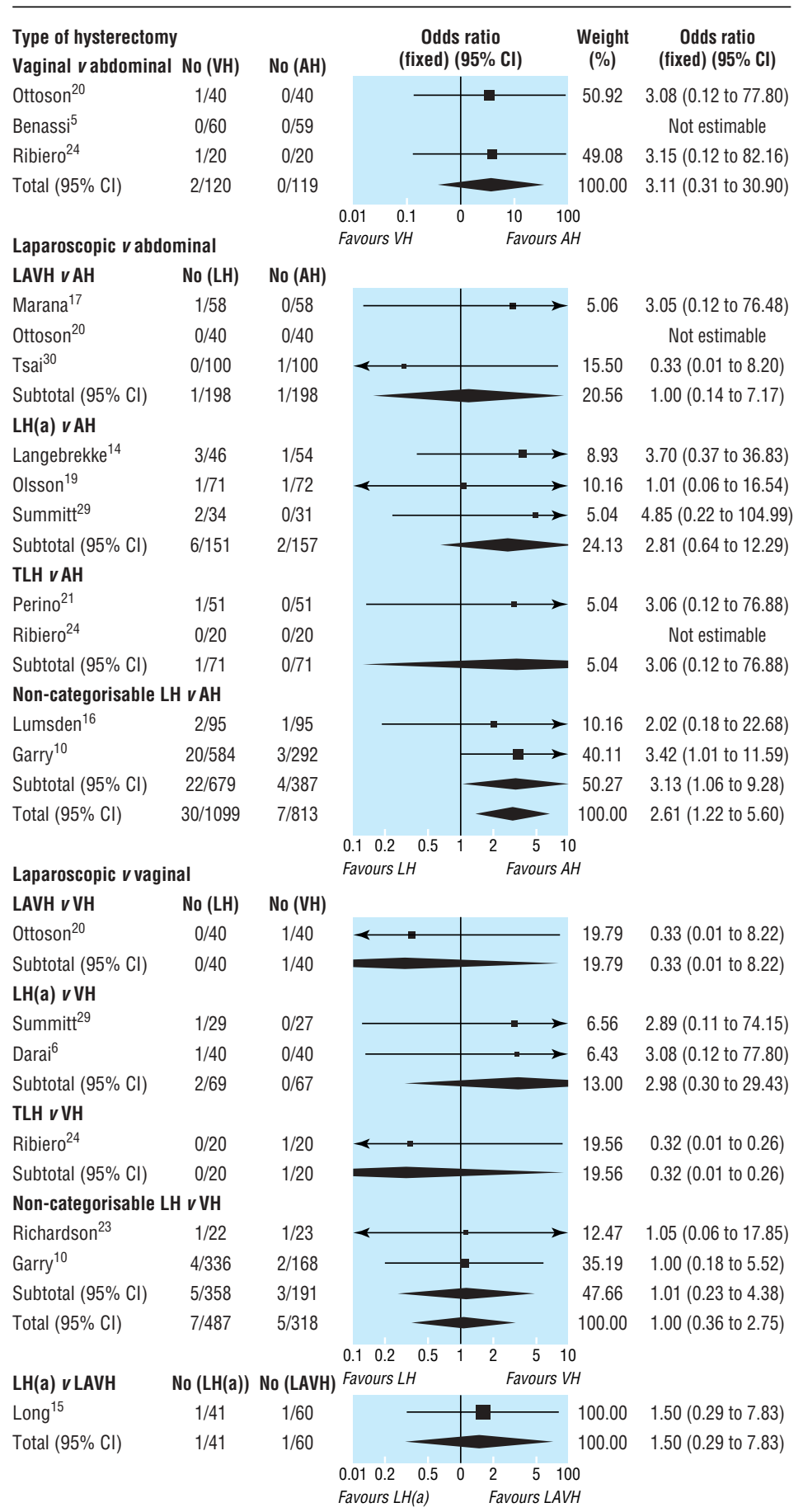

Figure 2 Meta-analysis of urinary tract (bladder or ureter) injury. Statistical pooling used fixed effects statistical model (no statistical heterogeneity present). $\mathrm{AH}=$ abdominal hysterectomy; $\mathrm{VH}=$ vaginal hysterectomy; $\mathrm{LH}=$ laparoscopic hysterectomy; $\mathrm{LAVH=laparoscopic}$ assisted vaginal hysterectomy; $\mathrm{LH}(\mathrm{a})=\mathrm{laparoscopic}$ hysterectomy where laparoscopic procedures include uterine artery ligation; TLH=total laparoscopic hysterectomy 


\section{What is already known on this topic}

Abdominal hysterectomy has been regarded as the most invasive approach to hysterectomy for women with benign disease

Laparoscopic hysterectomy requires greater surgical expertise

Previous reviews have reached different conclusions about the relative merits of laparoscopic, abdominal, and vaginal hysterectomy

\section{What this study adds}

Important gaps in outcome data have been highlighted, especially for long term outcomes

No outcomes are significantly worse for vaginal hysterectomy than for any other method of hysterectomy-vaginal is preferable to abdominal hysterectomy where possible

No evidence supports the use of laparoscopic hysterectomy rather than vaginal hysterectomy if the latter can be done safely

Compared with abdominal hysterectomy, laparoscopic hysterectomy is associated with less blood loss, shorter hospital stay, speedier return to normal activities, and fewer abdominal wall infections or febrile episodes, but it takes longer and urinary tract injuries are more likely

are compared (some surgeons are better trained in vaginal hysterectomy, and some in abdominal hysterectomy).

The method of hysterectomy in any given case will inevitably differ among gynaecologists. Until the past few years, the vast majority of hysterectomies for benign disease were still performed abdominally, ${ }^{3}$ and this is likely still to be the case in most settings. ${ }^{49}$ Although many gynaecologists in training are now exposed to laparoscopic hysterectomy, very few newly trained gynaecologists will have sufficient expertise and confidence to tackle total laparoscopic hysterectomy, which requires the highest level skills. More surgeons will be trained to do LAVH (and indeed some gynaecologists who have not received specific training have acquired the skills to perform LAVH and LH(a)). Although it has been suggested that LAVH does little more than combine the complications of laparoscopic surgery with those of vaginal surgery, ${ }^{3}$ this has not been supported in our review.

One important benefit of the introduction of LAVH and LH(a) into gynaecology training has been to increase surgeons' confidence and skill with vaginal surgery, thus making vaginal hysterectomy a more feasible option for many.

\section{Conclusions}

Our conclusions are limited by the strength of the evidence: for many outcomes, including many important long term outcomes, data were notably absent. Our review found no important disadvantages of vaginal hysterectomy compared with any other surgical approach, thus it remains an excellent option. Avoiding abdominal hysterectomy accelerates recovery, diminishes postoperative pain, and avoids abdominal wall infections and general postoperative febrile illness. Laparoscopic hysterectomy may help to avoid a laparotomy, but urinary tract injury is a genuine concern. Research is needed to ascertain longer term outcomes and to evaluate the newer approaches to hysterectomy, such as total laparoscopic hysterectomy.

We thank staff at the editorial base of the Cochrane Menstrual Disorders and Subfertility Group in Auckland, especially Cindy Farquhar and Michelle Proctor.

Contributors: NJ, DB, AL, and RG jointly conceived the idea for the study. $\mathrm{NJ}$ helped to design the study, wrote the protocol, helped to select trials for inclusion and do the data extraction, conducted the analyses, and wrote the final manuscript. He is also the guarantor for the paper. DB helped to design the study and commented on the protocol and the final manuscript. AL helped to design the study, commented on the protocol, helped with the selection of trials for inclusion, with data extraction, and with the analyses, and commented on the final manuscript. ET and LC helped with the selection of trials for inclusion, with data extraction, and with the analyses, and commented on the final manuscript. RG helped to design the study and commented on the protocol and the final manuscript.

Competing interests: RG is the principal investigator in a United Kingdom based multicentre randomised trial comparing laparoscopic hysterectomy with both abdominal and vaginal hysterectomy.

Ethical approval: Not needed.

1 Garry R, Reich H, Liu CY. Laparoscopic hysterectomy-definitions and indications. Gynaecol Endosc 1994;3:1-3.

Garry R. Towards evidence-based hysterectomy. Gynaecol Endosc 1998;7:225-33.

3 Reich H, Roberts L. Laparoscopic hysterectomy in current gynaecological practice. Rev Gynaecol Pract 2003;3:32-40.

4 Johnson N, Barlow D, Lethaby A, Tavender E, Curr E, Garry R. Surgical approach to hysterectomy for benign gynaecological disease. Cochrane Database Syst Rev 2005;(2):CD003677.

5 Benassi L, Rossi T, Kaihura CT, Ricci L, Bedocchi L, Galanti B. Abdominal or vaginal hysterectomy for enlarged uteri: a randomized clinical trial. Am J Obstet Gynecol 2002;187:1561-5.

6 Darai E, Soriano D, Kimata P, Laplace C, Lecuru F. Vaginal hysterectomy for enlarged uteri, with or without laparoscopic assistance: randomized study. Obstet Gynecol 2001;97:712-6.

7 Ellstrøm M, Olsen MF, Olsson J-H, Nordberg G, Bengtsson A, Hahlin M. Pain and pulmonary function following laparoscopic and abdominal hysterectomy: a randomized study. Acta Obstet Gynecol Scand 1998;77:923-8.

8 Falcone T, Paraiso MFR, Mascha E. Prospective randomized clinical trial of laparoscopically assisted vaginal hysterectomy versus total abdominal hysterectomy. Am J Obstet Gynecol 1999;180:955-62.

9 Ferrari MM, Berlanda N, Mezzopane R, Ragusa G, Cavallo M, Pardi G. Identifying the indications for laparoscopically assisted vaginal hysterectomy: a prospective, randomised comparison with abdominal hysterectomy in patients with symptomatic randomised comparison with abdominal hysterectomy
uterine fibroids. Br J Obstet Gynaecol 2000;107:620-5.

10 Garry R, Fountain J, Mason S, Hawe J, Napp V, Abbott J, et al. The eVALuate study: two parallel randomised trials, one comparing laparoscopic with abdominal hysterectomy, the other comparing laparoscopic with vaginal hysterectomy. BMJ 2004;328:129-38.

11 Härkki-Sirén P, Sjöberg J, Toivonen J, Tiitinen A. Clinical outcome and tissue trauma after laparoscopic and abdominal hysterectomy: a randomized controlled study. Acta Obstet Gynecol Scand 2000;79:866-71.

12 Hwang J-L, Seow K-M, Tsai Y-L, Huang L-W, Hsieh B-C, Lee C. Comparative study of vaginal, laparoscopically assisted vaginal and abdominal hysterectomies for uterine myoma larger than $6 \mathrm{~cm}$ in diameter or uterus weighing at least $450 \mathrm{~g}$ : a prospective randomized study. Acta Obstet Gynecol Scand 2002;81:1132-8.

13 Kunz G, Plath T, Leyendecker G. Comparison between laparoscopic assisted vaginal hysterectomy (LAVH) and abdominal hysterectomy. Geburtshilfe und Frauenheilkunde hysterectomy (LAV

14 Langebrekke A, Eraker R, Nesheim BI, Urnes A, Busund B, Sponland G. Abdominal hysterectomy should not be considered as a primary method for uterine removal-a prospective randomised study of 100 patients referred to hysterectomy. Acta Obstet Gynecol Scand 1996;75:404-7.

15 Long CY, Fang JH, Chen WC, Su JH, Hsu SC. Comparison of total laparoscopic hysterectomy and laparoscopically assisted vaginal hysterectomy. Gynecol Obstet Invest 2001;53:214-9.

16 Lumsden MA, Twaddle S, Hawthorn R, Traynor I, Gilmore D, Davis J, et al. A randomised comparison and economic evaluation of laparoscopic-assisted hysterectomy and abdominal hysterectomy. Br J Obstet Gynaecol 2000;107:1386-91.

17 Marana R, Busacca M, Zupi E, Garcea N, Paparella P, Catalano GF. Laparoscopically assisted vaginal hysterectomy versus total abdominal hysterectomy: a prospective, ranassisted vaginal hysterectomy versus total abdominal hysterecton
domized, multicenter study. Am J Obstet Gynecol 1999;180:270-5.

18 Miskry T, Magos A. Randomized, prospective, double-blind comparison of abdominal and vaginal hysterectomy in women without uterovaginal prolapse. Acta Obstet Gynecol Scand 2003;82:351-8.

19 Olsson J-H, Ellstrøm M, Hahlin M. A randomised prospective trial comparing laparoscopic and abdominal hysterectomy. Br J Obstet Gynaecol 1996;103:345-50.

20 Ottosen C, Lingman G, Ottosen L. Three methods for hysterectomy: a randomised, prospective study of short term outcome. Br J Obstet Gynaecol 2000;107:1380-5.

21 Perino A, Cucinella G, Venezia R, Castelli A, Cittadini E. Total laparoscopic hysterectomy versus total abdominal hysterectomy: an assessment of the learning curve in a prospective randomized study. Hum Reprod 1999;14:2996-9.

22 Raju KS, Auld BJ. A randomised prospective study of laparoscopic vaginal 2 Raju KS, Auld BJ. A randomised prospective study of laparoscopic vaginal hysterectomy versus abdominal hysterectomy

23 Richardson RE, Bournas N, Magos AL. Is laparoscopic hysterectomy a waste of time? Lancet 1995;345:36-41. 
24 Ribiero SC, Ribiero RM, Santos NC, Pinotti JA. A randomized study of total abdominal, vaginal and laparoscopic hysterectomy. Int J Gynecol Obstet 2003;83:37-43.

25 Schutz K, Possover M, Merker A, Michels A, Schneider A. Prospective randomize comparison of laparoscopic-assisted vaginal hysterectomy (LAVH) with abdominal hysterectomy $(\mathrm{AH})$ for the treatment of the uterus weighing $>200 \mathrm{~g}$. Surg Endosc 2002;16:121-5.

26 Seracchioli R, Venturoli S, Vianello F, Govoni F, Cantarelli M, Gualerzi B, et al. Total laparoscopic hysterectomy compared with abdominal hysterectomy in the presence of a large uterus. J Am Ass Gynecol Laparosc 2002;9:333-8.

27 Soriano D, Goldstein A, Lecuru F, Darai E. Recovery from vaginal hysterectomy compared with laparoscopy-assisted hysterectomy: a prospective, randomized, multicenter tudy Acta Obstet Gynecol Scand 2001;80:337-41.

28 Summitt RL Jr, Stovall TG, Lipscomb GH, Ling FW. Randomized comparison of laparoscopy-assisted vaginal hysterectomy with standard vaginal hysterectomy in an outpatient setting. Obstet Gynecol 1992;80:895-901.

29 Summitt RL Jr, Stovall TG, Steege JF, Lipscomb GH. A multicenter randomized comparison of laparoscopically assisted vaginal hysterectomy and abdominal hysterectomy in abdominal hysterectomy candidates. Obstet Gynecol 1998;92:321-6.

30 Tsai EM, Chen HS, Long CY, Yang CH, Hsu SC, Wu CH, et al. Laparoscopically assisted vaginal hysterectomy versus total abdominal hysterectomy: a study of 100 cases on light-endorsed transvaginal section. Gynecol Obstet Invest 2003;55:105-9.

31 Yuen PM, Mak TWL, Yim SF, Ngan Kee WD, Lam CWK, Rogers MS, et al Metabolic and inflammatory responses after laparoscopic and abdominal hysterectomy. Am J Obstet Gynecol 1998;179:1-5.

32 Apoola A, Hefni MA. Hysterectomy for moderately enlarged uterus: abdominal versus Apoola A, Hefni MA. Hysterectomy for moderately en

33 Chapron C, Laforest L, Ansquer Y, Fauconnier A, Fernandez B, Breart G, et al. Hysterectomy techniques used for benign pathologies: results of a French multicentre study. Hum Reprod 1999;14:2464-70

34 Ellstrom MA, Astrom M, Moller A, Olsson JH, Hahlin M. A randomized trial comparing changes in psychological well-being and sexuality after laparoscopic and abdominal hysterectomy. Acta Obstet Gynecol Scand 2003;82:871-5.

35 Holub Z, Jabor A, Kliment L, Voracek J, Lukac J. Comparison of two procedures for laparovaginal hysterectomy: a randomized trial. Eur J Obstet Gynecol 2000;90:31-6.

36 Howard FM, Sanchez R. A comparison of laparoscopically assisted vaginal hysterectomy and abdominal hysterectomy.J Gynecol Surg 1993:9:83-9.

37 Møller C, Kehlet H, Friland SG, Schouenborg LO, Lund C, Ottesen B. Fast track hysterectomy. Eur J Obstet Gynecol Reprod Biol 2001;98:18-22.

38 Nezhat F, Nezhat C, Gordon S, Wilkins E. Laparoscopic versus abdominal hysterectomy.J Reprod Med 1992;37:247-9.

39 Oscarson U, Nussler E. Laparoscopic or abdominal surgery-does it matter? Int J Obste Gynecol 2003;2:42.

40 Park NH, Kim JW, Seo CS, Kim SH, Song YS, Kang SB, et al. Comparison of two laparoscopic methods of hysterectomy. Int J Obstet Gynecol 2003;2:79.

41 Phipps JH, John M, Nayak S. Comparison of laparoscopically assisted vaginal hysterectomy and bilateral salpingo-oophorectomy with conventional abdominal hysterectomy and bilateral salpingo-oophorectomy. Br J Obstet Gynaecol 1993;100:698-700.
42 Cucinella G, Gugliotta G, Adile B. Total laparoscopic hysterectomy versus total abdominal hysterectomy: a confront of 100 versus 101 cases in prospective randomized study. In: XVI FIGO world congress abstract book 3. Washington, DC:2000:45

43 Davies A. Randomized controlled trial comparing oophorectomy at vaginal and laparoscopically assisted vaginal hysterectomy. Br J Obstetrics $\mathcal{E}$ Gynaecol 1998:poster abstracts.

$44 \mathrm{Hahlin}$ M, Ellstrom M, Olsson JH. Laparoscopic hysterectomy. Further knowledge of advantages and disadvantages is necessary. Lakartidningen 1994;91:220-2.

45 Pabuccu R, Atay V, Ergun A, Duru NK, Orhon E, Yenen MC. Laparoscopic assisted vaginal hysterectomy results. In: Human reproduction: abstracts of the 12th annual meeting of ESHRE. Vol 11. Maastricht: Oxford University Press, 1996:235-6.

46 Petrucco OM, Luke C, Moss J, Healy D. Randomized prospective trial comparing laparoscopic assisted vaginal and abdominal hysterectomy. In: IX annual scientific meeting of the Australian Grnaecological Endoscopic Society abstracts. Adelaide: Australian Gynaecological Endoscopy Society, 1999:75.

47 Garry R, Phillips G. How safe is the laparoscopic approach to hysterectomy? Gynaecol Endosc 1995;4:77-9.

48 Härkki-Sirén P, Sjoberg J, Makinen J. Finnish national register of laparoscopic hysterectomies: a review and complications of 1165 operations. Am J Obstet Gynaecol 1997;176:118-22.

49 Farquhar CM, Steiner CA. Hysterectomy rates in the United States 1990-1997. Obstet Gynecol 2002;99:229-34

(Accepted 9 May 2005)

bmj.com 2005;330:1478

University of Auckland, National Women's Department of Obstetrics and Gynaecology, Auckland Hospital, Auckland, New Zealand

Neil Johnson associate professor

Anne Lethaby biostatistician

Liz Curr registrar in obstetrics and gynaecology

Nuffield Department of Obstetrics and Gynaecology, John Radcliffe Hospital,

Oxford OX3 9DU

David Barlow head of department

Cochrane Oral Health Group, University of Manchester, Manchester

Emma Tavender collaborative review group coordinator

University of Western Australia Department of Obstetrics and Gynaecology, Perth, Australia

Ray Garry professor of obstetrics and gynaecology

Correspondence to: N Johnson n.johnson@auckland.ac.nz 